\title{
Advanced Computation Method for Double Core Hole Spectra : Insight Into the Nature of Intense Shake-up Satellites
}

\author{
Anthony Ferté* ${ }^{1}$ Jérôme Palaudoux, ${ }^{1}$ Francis Penent, ${ }^{1}$ Hiroshi Iwayama, ${ }^{2}$ Eiji Shigemasa, ${ }^{2}$ Yasumasa \\ Hikosaka, ${ }^{3}$ Kouichi Soejima, ${ }^{4}$ Kenji Ito, ${ }^{5}$ Pascal Lablanquie, ${ }^{1}$ Richard Taïeb,${ }^{1}$ and Stéphane Carniato ${ }^{*}$ \\ ${ }^{1}$ Laboratoire de Chimie Physique Matière et Rayonnement (LCPMR), \\ Sorbonne Université and CNRS, F-75005 Paris, France \\ ${ }^{2}$ UVSOR Facility, Institute for Molecular Science, Okazaki 444-8585, Japan \\ ${ }^{3}$ Institute of Liberal Arts and Sciences, University of Toyama, Toyama 930-0194, Japan \\ ${ }^{4}$ Department of Environmental Science, Niigata University, Niigata, 950-2181, Japan \\ ${ }^{5}$ Photon Factory, Institute of Materials Structure Science, Tsukuba 305-0801, Japan
}

(Dated: May 20, 2020)

E-mail : anthony.ferte@sorbonne-universite.fr ; stephane.carniato@upmc.fr 


\begin{abstract}
Double core hole spectroscopy is an ideal framework for investigating photoionization shake-up satellites. Their important intensity in a single site double core hole (ssDCH) spectrum allows the exploration of the subtle mix of relaxation and correlation effects associated with the inherent multielectronic character of the shake-up process. We present a high accuracy computation method for single photon double core shell photoelectron spectra that combines a selected configuration interaction procedure with the use of non orthogonal molecular orbitals to obtain unbiased binding energy and intensity. This strategy leads to oxygen ssDCH spectrum of the CO molecule that is in excellent agreement with the experimental result. Through a combined wave function and density analysis, we highlight that the most intense shake-up satellites are characterized by an electronic reorganization that opposes the core hole induced relaxation.
\end{abstract}

\title{
TOC graphic
}

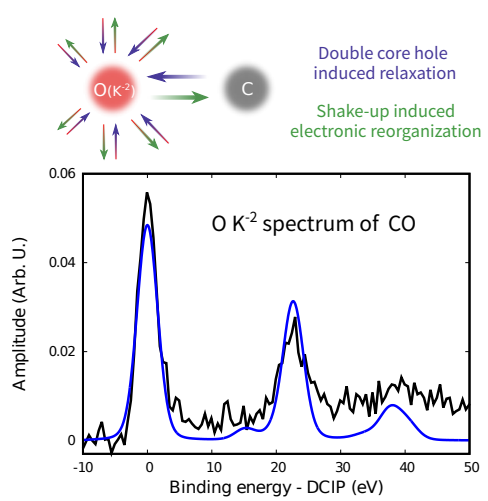

Keywords : Double core hole process; Multi-electron coincidence spectroscopy; CIPSI calculation; Non-orthogonal molecular orbitals; Shake-up satellite 
Shake-up satellite peaks are among the most interesting features in a photoelectron spectrum. Stemming from the conjunction of two processes, the photoionization and the excitation of the remaining bound electrons, they have an intrinsic multielectronic character and thus are associated with an intricate and subtle mix of relaxation and correlation effects.

Such satellites appear in many core electron photoionization processes that are the root of useful analysis methods. For instance, single core hole (SCH) X-ray Photoelectron Spectroscopy (XPS) [1-3] is one of the most applied techniques for chemical analysis as it is chemical specific and sensitive to the chemical environment.

Because of the peculiar nature of the satellites, interpretation of such peaks is a theoretical challenge and requires elaborated computational methods that goes beyond the mean field model of HartreeFock (HF) theory to reproduce both their energy and intensity. The sudden approximation, suggested by Bloch [4], was the first theoretical framework adopted to investigate XPS satellites [5-8], later extended by the dipole approximation [9] that allowed Martin and Shirley to develop a complete quantum model of SCH shake-up lines [10].

Thanks to experimental and theoretical development, double core shell photoionization had a continuously growing attractivity for the past decade. The current generation of X-ray sources finally allowed to observe the formation of doubly core ionized molecular system consecutive to either the interaction with a single X-ray photon, using syn- chrotron radiation [11], or to a sequential absorption of two X-ray photons, by mean of X-FEL radiation [12].

The early theoretical work of Cederbaum et. al. [13-15] showed that spectroscopies based on double core hole states display, in many cases, an enhanced sensitivity compared to SCH-XPS. In particular, for doubly core ionized system in which the two holes are located on the same atom, i.e. single site double core hole ( $\mathrm{ssDCH}$ or $\mathrm{K}^{-2}$ ) states, the satellite peaks are spread over a large energy range and it is not uncommon for the shake-up region to be responsible for more than half of the complete signal, while it amounts to only a few percent in SCH-XPS. This means that $\mathrm{ssDCH}$ spectra are drastically affected by the quality of the description of its satellite lines. Thus, one needs to take into account a large number $(\sim 50-100)$ of excited states and to provide a balanced description of transition moments to compute accurate ssDCH spectra. For these reasons, ssDCH spectroscopy is a stringent test subject for new theoretical developments.

In the context of SCH-XPS, many computation strategies were considered to investigate shake-up satellites [16-22, 24? -28]. However, since double core hole spectroscopy is a relatively new field, fewer methods have been proposed to reproduce ssDCH shake-up satellites [29-33].

We present a robust and unbiased method to compute accurate single photon $\mathrm{K}^{-2}$ satellite spectra. This approach relies on the combined use of a selected configuration interaction (CI) proce- 
dure, with the use of non orthogonal Molecular Orbital (MO) basis sets to describe the neutral and the ionized system and compute transition properties. We assess this method by computing the full oxygen ssDCH spectrum of the $\mathrm{CO}$ molecule and compare it with a new experimental spectrum with improved statistics in regard to the one previously published by some of the present authors and their co-workers [34]. Previous studies already investigated the $\mathrm{O} \mathrm{K}^{-2}$ spectrum of $\mathrm{CO}[32,34-36]$ but focused only on the double core ionization energy or yielded mitigated results on the satellites. Instead, our results display an excellent adequacy with experiment for both the position and the intensity of the main and shake-up lines. We also present a wave function and density analysis providing insight into the nature of the intense shakeup satellites.

Atomic units are used throughout this Letter if no specification is given.

In the dipole approximation, the amplitude of a peak associated with the formation of a ssDCH state through the absorption of a single X-ray photon is proportional to the squared transition moment between the initial $N$ electron neutral state wave function $\Psi_{\mathrm{I}}(N)$ and the final state wave function $\Psi_{\mathrm{F}}(N)=\hat{\mathcal{A}}(N) \mathcal{X}_{\mathbf{k}_{1}, \mathbf{k}_{2}}(1,2) \Psi_{\mathrm{F}}^{\mathrm{K}-2}(N-2)$ where $\hat{\mathcal{A}}(N)$ is the $N$ electron antisymetrizer, $\Psi_{\mathrm{F}}^{\mathrm{K}-2}(N-2)$ is the $(N-2)$ electron wave function describing the remaining bound electrons in the final ion and $\mathcal{X}_{\mathbf{k}_{1}, \mathbf{k}_{2}}(1,2)$ is a continuum wave function of two electrons of asymptotic momenta $\mathbf{k}_{1}$ and $\mathbf{k}_{2}$ respectively. Thus, in the length gauge, the differential cross section is given by

$$
\begin{aligned}
\frac{\mathrm{d}^{6} \sigma_{\mathrm{I} \rightarrow \mathrm{F}}}{\mathrm{d} \mathbf{k}_{1} \mathrm{~d} \mathbf{k}_{2}}=4 \pi \alpha \omega & \times\left|\left\langle\Psi_{\mathrm{F}}(N)\left|\sum_{i=1}^{N} \mathbf{r}_{i}\right| \Psi_{\mathrm{I}}(N)\right\rangle\right|^{2} \\
& \times \mathcal{L}\left(\omega-\left(E_{\mathrm{F}}+\epsilon\right) ; \Gamma_{\mathrm{F}}\right)
\end{aligned}
$$

where $\omega$ is the incident photon energy, $E_{\mathrm{F}}$ is the binding energy of the final $\mathrm{K}^{-2}$ state, $\epsilon$ is the total kinetic energy of the two photoelectrons and finally $\mathcal{L}\left(\omega-\left(E_{\mathrm{F}}+\epsilon\right) ; \Gamma_{\mathrm{F}}\right)$ is the Lorentzian function accounting for the lifetime of the final $\mathrm{ssDCH}$ state. One can show that the transition moment associated with a peak in the ssDCH spectrum is approximately [68]

$$
T_{\mathrm{I} \rightarrow \mathrm{F}} \propto\left\langle\Psi_{\mathrm{F}}^{\mathrm{K}-2}(N-2)\left|\hat{a}_{1 \mathrm{~s}}^{\alpha} \hat{a}_{1 \mathrm{~s}}^{\beta}\right| \Psi_{\mathrm{I}}(N)\right\rangle
$$

where $\hat{a}_{1 \mathrm{~s}}^{\alpha / \beta}$ is the annihilation operator that removes an electron from the targeted core spin orbital.

The initial neutral and final dication wave functions are obtained by CI calculations. To limit the number of determinants without favoring any state of the ion, we did not arbitrarily truncate the CI space. Instead, we used a selected CI procedure [37-42] called Configuration Interaction using a Perturbative Selection made Iteratively (CIPSI) that allows to iteratively build the wave function by only adding the most important Slater determinants. The variant used in this work is based on an iterative selection procedure using a second-order Epstein-Nesbet perturbation theory $[43,44]$ criterion and a semi-stochastic approach [45] to compute the perturbative second-order (PT2) energy (see Technical Details bellow and Ref. 46). We note that this CIPSI algorithm is tuned to balance the selection over all of the states considered.

The ssDCH wave functions are expanded as linear combination of Slater determinants constructed on the basis of relaxed $\mathrm{MO},\{v\}$, adapted to describe the core dication. These orbitals are obtained by reoptimization of the neutral HF orbitals [4750]. In this work, we used a partial reoptimization method, akin to Wood's suggestion [19], in which the double core hole orbital is kept frozen. Thus, the wave function of the $n^{\text {th }}$ ion state is

$$
\left|\Psi_{\mathrm{F}}^{\mathrm{K}-2, n}\right\rangle=\sum_{\nu} C_{\nu}^{n}\left|\varphi_{\nu}^{\mathrm{K}-2}\right\rangle
$$

where $\left|\varphi^{\mathrm{K}-2}\right\rangle=\left|v_{3}, v_{4}, \cdots, v_{N}\right\rangle$ are relaxed $N-2$ electron Slater determinants with an empty core orbital.

The most common choice is to use the same relaxed MOs to build the neutral wave function as it leads to easy computation of transition properties. However, these MOs are not adapted to describe the initial neutral system, so this leads to a slow convergence of the transition amplitude and energy. Instead, we used the standard neutral HF orbitals, $\{u\}$, to build the Slater determinants used in the expansion of the neutral system wave function:

$$
\left|\Psi_{\mathrm{I}}\right\rangle=\sum_{\mu} C_{\mu}\left|\phi_{\mu}\right\rangle
$$

where $|\phi\rangle=\left|1 \mathrm{~s}^{\alpha}, 1 \mathrm{~s}^{\beta}, u_{3}, \cdots, u_{N}\right\rangle$ are $N$ electron Slater determinants with a filled 1 s orbital. Introducing these expressions in (2) gives

$$
T_{\mathrm{I} \rightarrow \mathrm{F}, n}=\sum_{\mu} \sum_{\nu} C_{\mu} C_{\nu}^{n}\left\langle\varphi_{\nu}^{\mathrm{K}-2} \mid \phi_{\mu}^{\mathrm{K}-2}\right\rangle
$$

where $\left|\phi_{\mu}^{\mathrm{K}-2}\right\rangle=\hat{a}_{1 \mathrm{~s}}^{\alpha} \hat{a}_{1 \mathrm{~s}}^{\beta}\left|\phi_{\mu}\right\rangle$.

We used Löwdin's rules [51] to compute the overlap in equation (5). According to said rules, the overlap between two $(N-2)$ electron Slater determinants is

$$
\left\langle\varphi^{\mathrm{K}-2} \mid \phi^{\mathrm{K}-2}\right\rangle=\left|\begin{array}{cccc}
\left\langle v_{3} \mid u_{3}\right\rangle & \left\langle v_{3} \mid u_{4}\right\rangle & \cdots & \left\langle v_{3} \mid u_{N}\right\rangle \\
\left\langle v_{4} \mid u_{3}\right\rangle & \left\langle v_{4} \mid u_{4}\right\rangle & \cdots & \vdots \\
\vdots & \vdots & \ddots & \vdots \\
\left\langle v_{N} \mid u_{3}\right\rangle & \cdots & \cdots & \left\langle v_{N} \mid u_{N}\right\rangle
\end{array}\right|
$$



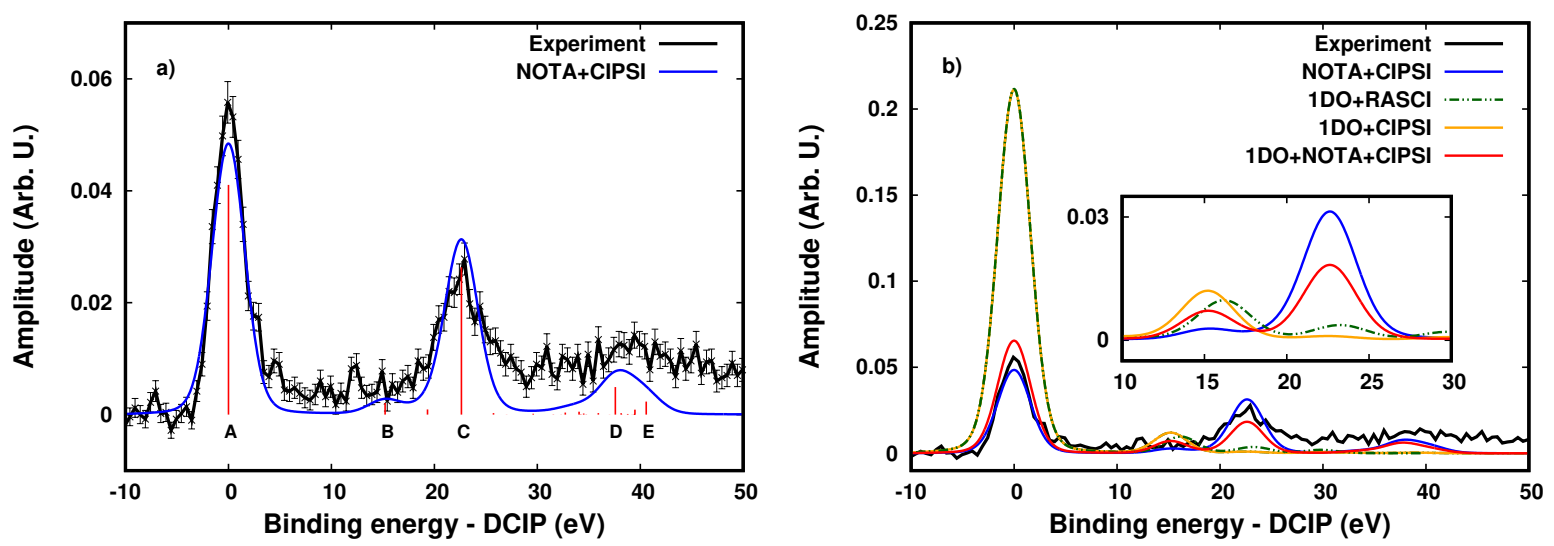

FIG. 1: a) Experimental (black) and computed NOTA+CIPSI (blue) $\mathrm{O} \mathrm{K}^{-2}$ spectra of CO. Vertical red lines are of size $\frac{1}{5} \times\left|T_{\mathrm{I} \rightarrow \mathrm{F}}\right|^{2}$. Experimental measurement uses three-electron coincidence events between the two photoelectrons and the Auger hypersatellite in the 505-605 eV energy range. The spectrum is obtained from the sum of the kinetic energies of the photoelectrons. Slow electrons (kinetic energy $<10 \mathrm{eV}$ ) have been excluded and the bining step of the histogram was set to $500 \mathrm{meV}$. Computation was done with the aug-cc-pVTZ basis set. The CIPSI calculations were iterated until convergence of transition amplitudes and relative binding energies. The final numbers of configurations in the neutral and ion wave functions were respectively around $4.5 \times 10^{6}$ and $3.4 \times 10^{6}$. The PT2 correction was used and the energy was shifted to set the DCIP to $0 \mathrm{eV}$. Only states with a transition amplitude superior to 0.01 were labeled. b) Comparison with, 1DO+CIPSI (orange), 1DO+NOTA+CIPSI (red) and Tashiro et. al. 1DO+RASCI [32] (dashed green). inset: Zoom on the 10 to $30 \mathrm{eV}$ shake-up region.

with $\left\langle v_{j} \mid u_{i}\right\rangle$ the overlap integrals between the two sets of occupied spin-orbitals.

We present in Fig. 1 our theoretical and experimental $\mathrm{O} \mathrm{K}^{-2}$ spectra of the $\mathrm{CO}$ molecule and we report in table I the binding energy and intensity, $\left|T_{\mathrm{I} \rightarrow \mathrm{F}}\right|^{2}$ (also called pole strength and spectroscopic factor $[52,53]$ ), of the most important peaks as well as the dominant configurations in the wave function of the associated final ssDCH state. The present experimental spectrum uses three-electron coincidence events between the two photoelectrons and an Auger hypersatellite detected via a magnetic bottle time-of-flight (MBTOF) electron spectrometer that was used in previous studies [34, 54-58]. A coincidence data set was accumulated with $\mathrm{CO}$ molecules at a photon energy of $1341 \mathrm{eV}$ for 14 hours, with an electron count rate reduced to 1500 electrons per second in order to minimize false coincidences. Electrons of less than $10 \mathrm{eV}$ have been excluded from the analysis to reduce background. More details about computation and experimental measurement are given in the Technical Details bellow.

The computation method proposed in this work involves a Non Orthogonal computation of Transition Amplitudes so we will refer to these results as $(\mathrm{NOTA}+\mathrm{WF})$, where WF stands for the wave function ansatz considered (CIPSI in this work). The absolute Double Core Ionization Potential (DCIP) was estimated to be $1177.51 \mathrm{eV}$ by an independent $\Delta$ CIPSI calculation corrected for relativistic effect by a third order Douglas-Kroll $(\mathrm{DK}(3))$ contribution (see Technical Details). This value is in good agreement with the experimental measurement of $1178.0 \pm 0.8 \mathrm{eV}$ [34]. The following discussion will focus on the general shape of the spectrum, thus, all spectra are shifted to set the DCIP at $0 \mathrm{eV}$.

Figure 1 displays a very good agreement between the NOTA+CIPSI and experimental spectra for both peak position and intensity. The $\mathrm{CO} \mathrm{O} \mathrm{K}^{-2}$ spectrum is characterized by three features. The principal peak corresponds, as intended, to the double photoionization of the two $\mathrm{O}$ 1s electrons followed by the complete relaxation of the outer shell electrons to the most stable $\mathrm{K}^{-2}$ state labeled A. The second feature is a satellite region of intensity comparable to that of the main line around 20 to $25 \mathrm{eV}$ with respect to the DCIP. We assigned this satellite region to one specific shake-up state labeled C. Finally, a less intense satellite region around 35 to $45 \mathrm{eV}$ is shown to stem from the transition to two shake-up states, D and E.

In an early work, Tashiro et. al. [32] attempted to compute the $\mathrm{O} \mathrm{K} \mathrm{K}^{-2}$ spectrum of $\mathrm{CO}$ by means of a Restricted Active Space (RAS) CI method. Nevertheless, they did not reproduce the spectrum correctly mainly because of a very crude approximation on the transition amplitude. Indeed, they considered a single determinant ansatz for the neutral wave function in the expression of the transition moment. In the following, we will refer to this approximation as the one determinant overlap (1DO) approximation. This approximation can be considered reasonable provided that one uses the correct MO basis set for the neutral system. How- 
TABLE I: NOTA+CIPSI transition amplitudes and relative binding energies associated with the intense peaks of the $\mathrm{O} \mathrm{K}^{-2}$ spectrum of $\mathrm{CO}$, and their corresponding final ssDCH state.

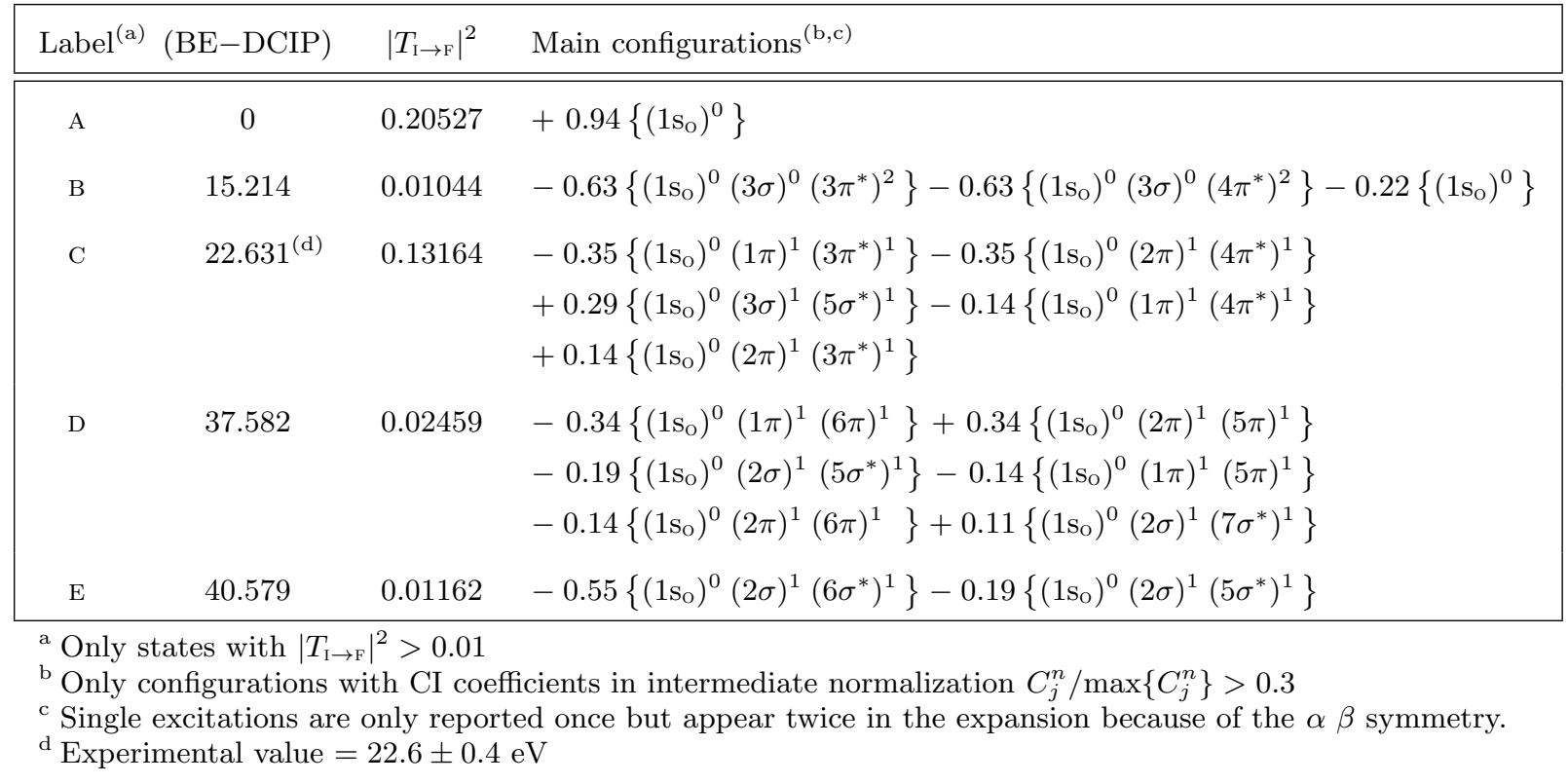

ever, they used the MO optimized for the $\mathrm{DCH}$ cation (equivalent to the $\{v\} \mathrm{MO}$ in this work) to describe the neutral system. In this context, the transition amplitude becomes

$$
T_{\mathrm{I} \rightarrow \mathrm{F}, n}=\left\langle\Psi_{\mathrm{F}}^{\mathrm{K}-2, n} \mid \varphi_{\mathrm{HF}}^{\mathrm{K}-2}\right\rangle=C_{\mathrm{HF}}^{\mathrm{K}-2, n}
$$

where $C_{\mathrm{HF}}^{\mathrm{K}-2, n}$ is the coefficient associated with the "HF $\mathrm{K}^{-2}$ determinant", $\left|\varphi_{\mathrm{HF}}^{\mathrm{K}-2}\right\rangle=\hat{a}_{1 \mathrm{~s}}^{\alpha} \hat{a}_{1 \mathrm{~s}}^{\beta}\left|\varphi_{\mathrm{HF}}\right\rangle$, in the expansion of the $\mathrm{K}^{-2}$ ion $n^{\text {th }}$ excited state wave function [Eq. (3)], with $\left|\varphi_{\mathrm{HF}}\right\rangle$ the $N$ electron $\mathrm{HF}$ Slater determinant in the relaxed $\{v\}$ basis.

Figure 1 (b) shows the comparison between our NOTA+CIPSI spectrum, 1DO+CIPSI spectrum and Tashiro et. al.'s 1DO+RASCI spectrum. We also report the spectrum obtained within the $1 \mathrm{DO}$ approximation but with the use of the adequate MO basis $\{u\}$ for the neutral wave function and refer to this result as $1 \mathrm{DO}+\mathrm{NOTA}+\mathrm{CIPSI}$.

As suggested before, the 1DO approximation completely fails to provide a good description of the transition amplitude if the correct MOs are not used for the neutral wave function. Both 1DO+RASCI and 1DO+CIPSI results display an overly exaggerated first peak while the transition amplitude to the state labeled $\mathrm{C}$, is dramatically minimized. One can also notice an overestimation of the smaller peak (see zoom) associated with the transition to the final state labeled B. These discrepancies can be easily understood as consequences of the 1DO approximation. Since only the $C_{\mathrm{HF}}^{\mathrm{K}-2, n}$ coefficient intervenes in the $1 \mathrm{DO}$ approximated amplitude [Eq. (7)], only $\mathrm{K}^{-2}$ states strongly weighted by the $\left|\varphi_{\mathrm{HF}}^{\mathrm{K}-2}\right\rangle$ determinant appear as bright states. From table I, one can see that it is the case for states A and B. On the contrary, $\mathrm{K}^{-2}$ states associated with a smaller $C_{\mathrm{HF}}^{\mathrm{K}-2, n}$, such as state $\mathrm{C}$, appear weaker on the computed spectrum.

By contrast, the NOTA+CIPSI calculation is much more accurate, even within the 1DO approximation. This is due to the better description of the neutral wave function provided by the one determinant ansatz, if the correct MOs are used, and to the reduced constraint on the transition moments thanks to the non orthogonality between the $\{u\}$ and $\{v\}$ basis. Indeed, one can easily see that the $1 \mathrm{DO}+\mathrm{NOTA}$ amplitude is

$$
T_{\mathrm{I} \rightarrow \mathrm{F}, n}=\sum_{j} C_{j}^{n}\left\langle\varphi_{j}^{\mathrm{K}-2} \mid \phi_{\mathrm{HF}}^{\mathrm{K}-2}\right\rangle .
$$

This clearly shows that the NOTA strategy provides a better description of the transition amplitude compared to a method based on the use of a single MO basis for the neutral and the ion.

Finally, one can also notice differences in satellites positions between CIPSI and RASCI results. Tashiro et. al. found that the binding energies of states B and C are respectively $16.2 \mathrm{eV}$ and $23.5 \mathrm{eV}$ with respect to the DCIP. On the other hand, the CIPSI calculation gave $15.2 \mathrm{eV}$ and $22.6 \mathrm{eV}$. Experimentally, the intense satellite line associated with $\mathrm{C}$ is measured at $22.6 \pm 0.4 \mathrm{eV}$ with respect to the main line, which is in excellent agreement with our CIPSI result. Even if the binding energy with respect to the DCIP is not a variational quantity, it is still expected that an unbalanced or unfully converged calculation would result in an overestimation of this relative binding energy. That is because the $\mathrm{K}^{-2}$ ground state is generally 

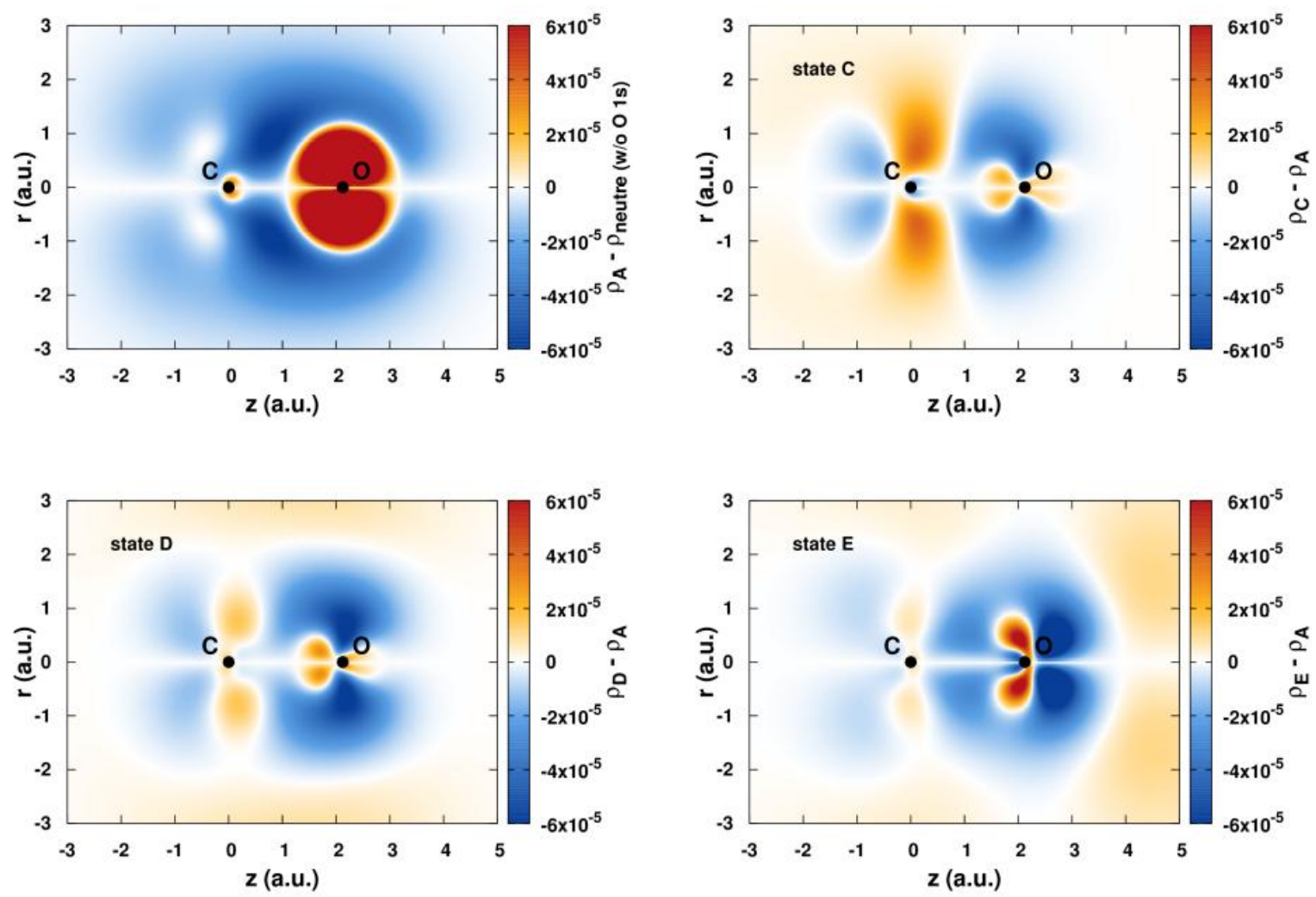

FIG. 2: Cylindrically integrated difference in density between top left panel) the unrelaxed $\mathrm{K}^{-2}$ state (the initial neutral system without the two $\mathrm{O} 1 \mathrm{~s}$ electrons) and the $\mathrm{K}^{-2}$ ground state A. top right panel ) the $\mathrm{K}^{-2}$ ground state A and shake-up state C. bottom left panel ) the $\mathrm{K}^{-2}$ ground state A and shake-up state D. bottom right panel ) the $\mathrm{K}^{-2}$ ground state A and shake-up state $\mathrm{E}$.

less affected by correlation effects than the shakeup states and so converges faster. This illustrates the robustness of the unbiased CIPSI method over an a priori truncated CI approach.

Due to the approximated monopolar nature of the transition moment [Eq. (2)], understanding why a ssDCH state leads to a bright transition is a quite straightforward task. It means that the more the $\mathrm{K}^{-2}$ state will be comparable to the neutral system, the more intense the transition will be.

The principal difference between the neutral system and the $\mathrm{K}^{-2}$ cation is the strong relaxation undergone by the outer shell electrons due to the increase in the effective nuclear charge. We report, in the top left panel of Fig. 2, the difference in electron density $\rho$ between the unrelaxed ssDCH (the neutral system from which we removed the two $\mathrm{O}$ 1s electrons) and the fully relaxed $\mathrm{K}^{-2}$ ground state A. The large positive (red) area around the $\mathrm{O}$ clearly illustrates the important shrinking of the electron density around the ionized atom. To quantify this relaxation, we integrate the complete positive (identically negative) component of the previous density difference. We obtained that the complete relaxation of the electron density in response to the ssDCH corresponds to a displacement of $\approx 2.07$ electrons. Analogous illustrations of the relaxation consecutive to double core ionization can be found in Refs. 35, 59-61 and to single core hole shake-up in Ref. 62.

The main $\mathrm{K}^{-2}$ state $\mathrm{A}$ is strongly dominated by the $\left|\varphi_{\mathrm{HF}}^{\mathrm{K}-2}\right\rangle$ determinant. Thus, it still holds some important similarities with the neutral system despite the relaxation. A good indicator is the comparison between the relaxed $\left|\varphi_{\mathrm{HF}}^{\mathrm{K}-2}\right\rangle$ and unrelaxed $\left|\phi_{\mathrm{HF}}^{\mathrm{K}-2}\right\rangle$ determinants. We found that the overlap between these two is close to 0.59 , meaning that a state dominated by $\left|\varphi_{\mathrm{HF}}^{\mathrm{K}-2}\right\rangle$ remains fairly similar to the neutral system and so gives rise to a bright line in the spectrum. The same argument explains the relative intensity of the transition to B. Indeed, one can see that $\left|\varphi_{\mathrm{HF}}^{\mathrm{K}-2}\right\rangle$ also plays a significant role in the wave function expansion of state $\mathrm{B}$, which differs from state A mainly by correlation effects, as shown by the important coefficient associated with double excitations.

Even though state $\mathrm{C}$ does not have the $\left|\varphi_{\mathrm{HF}}^{\mathrm{K}-2}\right\rangle$ determinant as a principal contribution to its wave function, it is still very bright suggesting that another effect should be responsible for its intensity. 
The wave function characterizing state $\mathrm{C}$ is heavily marked by important single excitations from bonding to antibonding orbitals and, more specifically, by excitation from $\pi$ to $\pi^{*}$ orbitals (see Table I). These single excitations are the signature of electronic relaxation. Since the bonding orbitals tend to be more localized on the $\mathrm{O}$, while the antibonding orbitals tend to have a significant $\mathrm{C}$ character, state $\mathrm{C}$ mainly differs from state A by relaxation of the electron density from the oxygen atom toward the carbon atom. The top right panel of Fig. 2 shows, the difference in electron density between state A and state C. This figure displays an important positive region around the $\mathrm{C}$ atom and a negative region around the $\mathrm{O}$ illustrating the transfer of electron density from the oxygen to the carbon characteristic of this shake-up state. The relaxation of the electron density toward the $\mathrm{C}$ atom, related to the displacement of $\approx 0.49$ electron, counterbalances the localization of the electron density around the $\mathrm{O}$ atom, resulting in the high intensity transition associated with this state.

Relaxation effects are also the main reason for the brightness of states D and E. Both wave functions describing these states are dominated by single excitations from tight bonding to more diffuse orbitals. The differences in electron density between state $\mathrm{A}$ and states $\mathrm{D}$ and $\mathrm{E}$ are reported in the bottom panels of Fig. 2. Both figures show that these two states are characterized by an overall expansion of the electron density illustrated by the negative region in the vicinity of the molecule counterbalanced by the vast positive region farther away. It corresponds to displacements of $\approx 0.80$ and $\approx 0.90$ electron for states $\mathrm{D}$ and $\mathrm{E}$, respectively. This inflation once again mitigates the contraction of the electron density resulting in the brightness of these states.

The effect of electronic relaxation on the relative intensity of core photoelectron shake-up satellites has already been reported $[29,31]$ and can be considered as very general. In particular, it explains why the shake-up intensity compared to the main line is much more important in ssDCH spectra than in SCH-XPS spectra. That is because the electronic relaxation effects are much stronger in a ssDCH than in a $\mathrm{SCH}$ system (see e.g. ref. 63). A similar reasoning based on the electronic reorganization associated with a given excited configuration already proved to be useful in understanding the modification of the $\mathrm{CO}$ SCH shake-up satellite spectrum depending on the atom bearing the core vacancy [64]. With our analysis, we give a clear illustration of the relation between relaxation and shake-up intensity, we quantify the magnitude of the electron density reorganization and we highlight a characteristic electronic effect associated with the high intensity shake-up satellites.

To summarize, we presented a high accuracy com- putation method for single photon double coreshell photoelectron satellites called NOTA+CIPSI. This strategy combines a selected CI wave function calculation with the use of two non orthogonal MO basis sets respectively optimized for the initial neutral and the final core ionized system.

This method was used to compute the $\mathrm{O}$ ssDCH spectrum of the $\mathrm{CO}$ molecule with near perfect adequacy to the experimental result. We give the credit for the NOTA+CIPSI accuracy to the combined usage of the CIPSI method, which provides a good and balanced description of all of the final ssDCH states, with our NOTA approach involving non orthogonal molecular orbital basis sets, which allows the use of the adequate MOs for both initial and final states and thus provides a good description of transition amplitudes. Being robust and accurate, the NOTA+CIPSI approach can be used as a direct mean of studying inner-shell photoelectron satellites as well as a reference material to discuss the results obtained by other methods.

We also combined wave function and density analysis to extract general information about the nature of intense satellites lines. In particular, we showed that bright shake-up states are marked by excited configurations inducing an electronic reorganization that opposes the contraction of the electron density due to the core hole. In summary, we highlight that bright shake-up states are the ones characterized by electronic effects that compensates the core hole induced relaxation.

\section{Technical details}

The NOTA+CIPSI calculations were done with the software QUANTUM PACKAGE 2.0 [46]. We implemented in this software all tools necessary to compute core hole spectra including the routines to use non-orthogonal MO basis sets.

We computed the $\mathrm{O} \mathrm{K}^{-2}$ spectrum of the $\mathrm{CO}$ molecule using Dunning aug-cc-pVTZ basis set $[65,66]$. The neutral wave function was computed within the frozen core approximation while the double core hole was restricted to the $\mathrm{O} 1 \mathrm{~s}$ orbital in the ion wave functions by virtue of the core valence separation approximation [15]. The CIPSI procedures were iterated until stabilization of both transition amplitudes and relative ionization energies. The final numbers of configuration were roughly $4.5 \times 10^{6}$ and $3.4 \times 10^{6}$ in the neutral and ion wave functions respectively.

The final spectrum was built using Lorentzian functions of area $\left|T_{\mathrm{I} \rightarrow \mathrm{F}}\right|^{2}$ and width $\Gamma_{\mathrm{F}}=0.5 \mathrm{eV}$ centered at the PT2 corrected ionization energy corresponding to the final state considered. Convolution with Gaussian functions of full width at half maximum equal to $3.5 \mathrm{eV}$ was used according to the resolution of the spectrometer. 
We did not try to include vibronic effects and relativistic correction was neglected on the computed spectrum as it is admitted that it would only have a small differential effect between the lines. However, relativistic correction is not negligible on the absolute DCIP. We estimated it to be $0.84 \mathrm{eV}$ from a $\triangle$ CISD (CI of single and double excitation) corrected by the third order Douglas-Kroll $(\mathrm{DK}(3))$ transformation method performed via the GAMESS(US) software [67].

The difference of density maps were obtained by angular integration on a cylindrical basis $\{r, \theta, z\}$. The $\mathrm{z}$ axis was chosen to coincide with the $\mathrm{CO}$ bond and the $r$ and $z$ coordinates were discretized on a $(401 \times 401)$ point grid.

We now present the CIPSI algorithm used in this work [46]. We start with a guess wave function $\left|\Psi_{0}\right\rangle=\sum_{\mathrm{A} \in \mathcal{R}} C_{\mathrm{A}}|\mathrm{A}\rangle$ where $|\mathrm{A}\rangle$ are Slater determinants of the initial guess CI space $\mathcal{R}$. Epstein-Nesbet multireference perturbation theory is used to predict the importance of a determinant $|\mathrm{B}\rangle \notin \mathcal{R}$. The second-order correction to the guess wave function energy $\left(E_{0}\right)$ stemming from $|\mathrm{B}\rangle$ is given by the relation

$$
\mathcal{E}_{\mathrm{B}}^{(2)}=\frac{\left|\left\langle\Psi_{0}|\hat{H}| \mathrm{B}\right\rangle\right|^{2}}{E_{0}-\langle\mathrm{B}|\hat{H}| \mathrm{B}\rangle} .
$$

The determinants associated with the largest corrections are then added to the CI space $\mathcal{R}$ and we iterate. Finally the energy is corrected by the residual second-order perturbation energy :

$$
\mathrm{E}+\mathrm{PT} 2=E_{0}+\sum_{\mathrm{B}} \mathcal{E}_{\mathrm{B}}^{(2)} .
$$

In this work, we used, as an initial guess, the CISD wave function to start the selection with a decent representation of the $\mathrm{K}^{-2}$ excited states.
The experimental measurements were performed on the BL-16 undulator beamline at the Photon Factory operated in top up, single bunch mode. An asynchronous mechanical chopper [55] was used to let through the light emitted by the single bunch only every $12.5 \mathrm{\mu s}$. This avoids the piling up of electrons produced by the raw single bunch emitting light pulses every $624 \mathrm{~ns}$, and makes it possible to measure absolute electron times of flight. The $2.5 \mathrm{~m}$ long MB-TOF spectrometer allows the detection of electrons of kinetic energy $\epsilon$ with very high efficiency $(70 \pm 5 \%$ with $\epsilon$ below $200 \mathrm{eV}$, $50 \pm 5 \%$ for $\epsilon$ around $500 \mathrm{eV}$ ) [54], and provides an energy resolution of $(\Delta \epsilon / \epsilon)=1.6 \%$. Time of flight to kinetic energy conversion is determined using $\operatorname{Ar} 2 \mathrm{p}$ photoelectrons at different kinetic energies after calibration of the photon energy on wellknown atomic and molecular $\left(\mathrm{Ar}, \mathrm{O}_{2}, \mathrm{Ne}\right)$ innershell resonances.

\section{Acknowledgments}

We thank Labex MiChem part of French state funds managed by the ANR within the Investissements d'Avenir programme (Sorbonne Université, ANR-11-IDEX-0004-02), that provided PhD funding for A. Ferté.

We are grateful to the Photon Factory staff for the stable operation of the storage ring. This work was performed with the approval of the PF Program Advisory Committee (Proposal No. 2010G621).

K. Soejima and K. Ito acknowledge the support of the Labex Plas@Par, managed by the Agence Nationale de la Recherche as part of the Programme d'Investissements d'Avenir under Reference No. ANR-11-IDEX-0004-02.

\section{References}

[1] C. Nordling, E. Sokolowski, and K. Siegbahn, "Precision method for obtaining absolute values of atomic binding energies," Phys. Rev., vol. 105, no. 5, pp. 1676-1677, 1957.

[2] E. Sokolowski, C. Nordling, and K. Siegbahn, "Chemical shift effect in inner electronic levels of $\mathrm{Cu}$ due to oxidation," Phys. Rev., vol. 110, no. 3, pp. 776-776, 1958.

[3] K. Siegbahn, C. Nordling, A. Fahlman, R. Nordberg, K. Hamerin, J. Hedman, G. Johansson, T. Bergmark, S. E. Karlsson, I. Lindgren, and B. Lindberg, "ESCA, atomic, molecular and solid state structure studied by means of electron spectroscopy," tech. rep., Almqvist and Wiksell, Uppsala, 1967.

[4] F. Bloch, "Double electron transitions in x-ray spectra," Phys. Rev., vol. 48, no. 3, pp. 187-192, 1935.

[5] T. Åberg, "Theory of x-ray satellites," Phys. Rev., vol. 156 , no. 1 , pp. 35-41, 1967.

[6] H. W. Meldner and J. D. Perez, "Observability of rearrangement energies and relaxation times," Phys. Rev. A: At., Mol., Opt. Phys., vol. 4, no. 4, pp. 1388-1396, 1971.

[7] L. J. Aarons, M. F. Guest, and J. H. Hillier, "Interpretation of high energy photoelectron spectra satellite peaks by semi-empirical molecular orbital calculations," J. Chem. Soc. Faraday Trans. 2, vol. 68, pp. 1866-1871, 1972.

[8] M. A. Brisk and A. D. Baker, "Shake-up satellites in x-ray photoelectron spectroscopy," J. Electron Spectrosc. Relat. Phenom., vol. 7, no. 3, pp. 197213, 1975.

[9] A. Rosencwaig, G. K. Wertheim, and H. J. 
Guggenheim, "Origins of satellites on inner-shell photoelectron spectra," Phys. Rev. Lett., vol. 27, no. 8, pp. 479-481, 1971.

[10] R. L. Martin and D. A. Shirley, "Theory of corelevel photoemission correlation state spectra," $J$. Chem. Phys., vol. 64, no. 9, pp. 3685-3689, 1976.

[11] P. Lablanquie, F. Penent, and Y. Hikosaka, "Multi-electron coincidence spectroscopy: double photoionization from molecular inner-shell orbitals," J. Phys. B, vol. 49, no. 18, p. 182002, 2016.

[12] N. Berrah and L. Fang, "Chemical analysis: Double core-hole spectroscopy with free-electron lasers," J. Electron Spectrosc. Relat. Phenom., vol. 204, pp. 284-289, 2015.

[13] L. S. Cederbaum, F. Tarantelli, A. Sgamellotti, and J. Schirmer, "On double vacancies in the core," J. Chem. Phys., vol. 85, no. 11, pp. 65136523, 1986.

[14] L. S. Cederbaum, F. Tarantelli, A. Sgamellotti, and J. Schirmer, "Double vacancies in the core of benzene," J. Chem. Phys., vol. 86, no. 4, pp. 21682175, 1987.

[15] L. S. Cederbaum, "Many-body theory of multiple core holes," Phys. Rev. A: At., Mol., Opt. Phys., vol. 35, no. 2, pp. 622-631, 1987.

[16] L. J. Aarons, M. Barber, M. F. Guest, I. H. Hillier, and J. H. McCartney, "Satellite peaks in the high energy photoelectron spectra of some small first row molecules," Mol. Phys., vol. 26, no. 5, pp. 1247-1256, 1973.

[17] V. Carravetta, H. Ågren, D. Nordfors, and S. Svensson, "Static exchange and Stieltjes imaging calculations; Interpretation of a new highresolution $\mathrm{Cl} 2 \mathrm{p}$ shake-up/shake-off spectrum of $\mathrm{HCl}, "$ Chem. Phys. Lett., vol. 152, no. 2-3, pp. 190-195, 1988.

[18] M. F. Guest, I. H. Hillier, V. R. Saunders, and M. H. Wood, "The theoretical description of ionic states observed by high energy photoelectron spectroscopy," Proc. R. Soc. A, vol. 333, no. 1593, pp. 201-215, 1973.

[19] M. H. Wood, "On the calculation of the satellite peaks observed in the high energy photoelectron spectra of small molecules," Chem. Phys., vol. 5, no. 3, pp. 471-477, 1974.

[20] R. L. Martin and D. A. Shirley, "Theory of the Neon 1s correlation-peak intensities," Phys. Rev. A: At., Mol., Opt. Phys., vol. 13, no. 4, pp. 1475$1483,1976$.

[21] M. F. Guest, W. R. Rodwell, T. Darko, I. H. Hillier, and J. Kendrick, "Configuration interaction calculations of the satellite peaks associated with C 1s ionization of carbon monoxide," $J$. Chem. Phys., vol. 66, no. 12, pp. 5447-5452, 1977.

[22] H. Basch, "Theoretical models for the interpretation of E.S.C.A. spectra," J. Electron Spectrosc. Relat. Phenom., vol. 5, no. 1, pp. 463-500, 1974.

[23] D. Nordfors, A. Nilsson, N. Mårtenson, S. Svensson, U. Gelius, and H. Ågren, "X-ray excited photoelectron spectra of free molecules containing Oxygen," J. Electron Spectrosc. Relat. Phenom., vol. 56, no. 2, pp. 117-164, 1991.

[24] S. Carniato, G. Dufour, F. Rochet, H. Roulet, P. Chaquin, and C. Giessner-Prettre, "Theory assisted interpretation of copper phthalocyanine core levels XPS spectra," J. Electron Spectrosc. Relat. Phenom., vol. 67, no. 1, pp. 189-209, 1994.

[25] G. Angonoa, O. Walter, and J. Schirmer, "Theoretical K-shell ionization spectra of $\mathrm{N} 2$ and $\mathrm{CO}$ by a fourth order Greens function method," J. Chem. Phys., vol. 87, no. 12, pp. 6789-6801, 1987.

[26] S. Coriani and H. Koch, "Communication: Xray absorption spectra and core-ionization potentials within a core-valence separated coupled cluster framework," J. Chem. Phys., vol. 143, no. 18, p. 181103, 2015.

[27] S. P. Neville and M. S. Schuurman, "A general approach for the calculation and characterization of x-ray absorption spectra," J. Chem. Phys., vol. 149, no. 15, p. 154111, 2018.

[28] B. Brena, Y. Luo, M. Nyberg, S. Carniato, K. Nilson, Y. Alfredsson, J. Åhlund, N. Mårtensson, H. Siegbahn, and C. Puglia, "Equivalent core-hole time-dependent density functional theory calculations of carbon 1s shake-up states of phthalocyanine," Phys. Rev. B: Condens. Matter Mater. Phys., vol. 70, no. 19, p. 195214, 2004.

[29] E. M.-L. Ohrendorf, L. S. Cederbaum, and F. Tarantelli, "Double vacancies in the cores of silane and tetrafluorosilane," Phys. Rev. A: At., Mol., Opt. Phys., vol. 44, no. 1, pp. 205-217, 1991.

[30] R. Santra, N. V. Kryzhevoi, and L. S. Cederbaum, "X-ray two-photon photoelectron spectroscopy: A theoretical study of inner-shell spectra of the organic para-aminophenol molecule," Phys. Rev. Lett., vol. 103, no. 1, p. 013002, 2009.

[31] N. V. Kryzhevoi, R. Santra, and L. S. Cederbaum, "Inner-shell single and double ionization potentials of aminophenol isomers," J. Chem. Phys., vol. 135 , no. 8, p. 084302, 2011.

[32] M. Tashiro, K. Ueda, and M. Ehara, "Double corehole correlation satellite spectra of $\mathrm{N}_{2}$ and $\mathrm{CO}$ molecules," Chem. Phys. Lett., vol. 521, pp. 4551, 2012.

[33] S. Carniato, P. Selles, A. Ferté, N. Berrah, A. H. Wuosmaa, M. Nakano, Y. Hikosaka, K. Ito, M. Žitnik, K. Bučar, L. Andric, J. Palaudoux, F. Penent, and P. Lablanquie, "Double-core ionization photoelectron spectroscopy of $\mathrm{C}_{6} \mathrm{H}_{6}$ : Breakdown of the intuitive ortho -meta -para binding energy ordering of $\mathrm{K}^{-1} \mathrm{~K}^{-1}$ states," J. Chem. Phys., vol. 151, no. 21, p. 214303, 2019.

[34] P. Lablanquie, F. Penent, J. Palaudoux, L. Andric, P. Selles, S. Carniato, K. Bučar, M. Žitnik, M. Huttula, J. H. D. Eland, E. Shigemasa, K. Soejima, Y. Hikosaka, I. H. Suzuki, M. Nakano, and K. Ito, "Properties of hollow molecules probed by single-photon double ionization," Phys. Rev. Lett., vol. 106, no. 6, p. 063003, 2011.

[35] M. Tashiro, M. Ehara, H. Fukuzawa, K. Ueda, C. Buth, N. V. Kryzhevoi, and L. S. Cederbaum, "Molecular double core hole electron spectroscopy for chemical analysis," J. Chem. Phys., vol. 132, no. 18, p. 184302, 2010.

[36] J. Lee, D. W. Small, and M. Head-Gordon, "Excited states via coupled cluster theory without equation-of-motion methods: Seeking higher roots with application to doubly excited states and double core hole states," J. Chem. Phys., vol. 151, no. 21, p. 214103, 2019. 
[37] C. F. Bender and E. R. Davidson, "Studies in configuration interaction: The first-row diatomic hydrides," Phys. Rev., vol. 183, p. 23, 1969.

[38] B. Huron, J. P. Malrieu, and P. Rancurel, "Iterative perturbation calculations of ground and excited state energies from multiconfigurational zeroth order wavefunctions," J. Chem. Phys., vol. 58, p. 5745, 1973.

[39] R. J. Buenker and S. D. Peyerimhoff, "Individualized configuration selection in CI calculations with subsequent energy extrapolation," Theor. Chim. Acta, vol. 35, p. 33, 1974.

[40] R. J. Buenker, S. D. Peyerimhoff, and P. J. Bruna, Comparative Theoretical Organic Chemistry. Dordrecht, The Netherlands: Reidel, 1981.

[41] S. Evangelisti, J.-P. Daudey, and J.-P. Malrieu, "Convergence of an improved CIPSI algorithm," Chem. Phys., vol. 75, no. 1, pp. 91 - 102, 1983.

[42] R. J. Harrison, "Approximating full configuration interaction with selected configuration interaction and perturbation theory," J. Chem. Phys., vol. 94, no. 7, p. 5021, 1991.

[43] P. S. Epstein, "The Stark effect from the point of view of Schroedinger's quantum theory," Phys. Rev., vol. 28, no. 4, pp. 695-710, 1926.

[44] R. K. Nesbet, "Configuration interaction in orbital theories," Proc. R. Soc. A, vol. 230, no. 1182, pp. 312-321, 1955.

[45] Y. Garniron, A. Scemama, P.-F. Loos, and M. Caffarel, "Hybrid stochastic-deterministic calculation of the second-order perturbative contribution of multireference perturbation theory," $J$. Chem. Phys., vol. 147, no. 3, p. 034101, 2017.

[46] Y. Garniron, T. Applencourt, K. Gasperich, A. Benali, A. Ferté, J. Paquier, B. Pradines, R. Assaraf, P. Reinhardt, J. Toulouse, P. Barbaresco, N. Renon, G. David, J.-P. Malrieu, M. Véril, M. Caffarel, P.-F. Loos, E. Giner, and A. Scemama, "Quantum Package 2.0: An OpenSource Determinant-Driven Suite of Programs," J. Chem. Theory Comput., vol. 15, no. 6, pp. 35913609, 2019.

[47] P. S. Bagus, "Self-Consistent-Field wave functions for hole states of some Ne-like and Ar-like ions," Phys. Rev., vol. 139, no. 3A, pp. A619-A634, 1965.

[48] H. J. A. Jensen, P. Jørgensen, and H. Ågren, "Efficient optimization of large scale MCSCF wave functions with a restricted step algorithm," $J$. Chem. Phys., vol. 87, no. 1, pp. 451-466, 1987.

[49] H. Ågren and H. J. A. Jensen, "Relaxation and correlation contributions to molecular double core ionization energies," Chem. Phys., vol. 172, no. 1, pp. $45-57,1993$.

[50] A. T. B. Gilbert, N. A. Besley, and P. M. W. Gill, "Self-consistent field calculations of excited states using the maximum overlap method (MOM)," J. Phys. Chem. A, vol. 112, no. 50, pp. 13164-13171, 2008.

[51] P.-O. Löwdin, "Quantum theory of many-particle systems. I. Physical interpretations by means of density matrices, natural spin-orbitals, and convergence problems in the method of configurational interaction," Phys. Rev., vol. 97, no. 6, pp. 1474-1489, 1955.

[52] L. S. Cederbaum, W. Domcke, J. Schirmer, and
W. Von Niessen, "Many-body effects in valence and core photoionization of molecules," Phys. Scr., vol. 21, no. 3-4, pp. 481-491, 1980.

[53] L. S. Cederbaum, W. Domcke, J. Schirmer, and W. Von Niessen, Correlation Effects in the Ionization of Molecules: Breakdown of the Molecular Orbital Picture, vol. LXV, pp. 115-159. John Wiley \& Sons, 1986.

[54] P. Lablanquie, T. P. Grozdanov, M. Žitnik, S. Carniato, P. Selles, L. Andric, J. Palaudoux, F. Penent, H. Iwayama, E. Shigemasa, Y. Hikosaka, K. Soejima, M. Nakano, I. H. Suzuki, and K. Ito, "Evidence of single-photon two-site core double ionization of $\mathrm{C}_{2} \mathrm{H}_{2}$ molecules," Phys. Rev. Lett., vol. 107, no. 19, p. 193004, 2011.

[55] M. Nakano, F. Penent, M. Tashiro, T. P. Grozdanov, M. Žitnik, S. Carniato, P. Selles, L. Andric, P. Lablanquie, J. Palaudoux, E. Shigemasa, H. Iwayama, Y. Hikosaka, K. Soejima, I. H. Suzuki, N. Kouchi, and K. Ito, "Single photon $\mathrm{K}^{-2}$ and $\mathrm{K}^{-1} \mathrm{~K}^{-1}$ double core ionization in $\mathrm{C}_{2} \mathrm{H}_{2 \mathrm{n}}(\mathrm{n}=1$ 3 ), $\mathrm{CO}$, and $\mathrm{N}_{2}$ as a potential new tool for chemical analysis," Phys. Rev. Lett., vol. 110, no. 16, p. 163001, 2013.

[56] K. Ito, F. Penent, Y. Hikosaka, E. Shigemasa, I. H. Suzuki, J. H. D. Eland, and P. Lablanquie, "Application of a simple asynchronous mechanical light chopper to multielectron coincidence spectroscopy," Rev. Sci. Instrum., vol. 80, no. 12, p. 123101, 2009.

[57] Y. Hikosaka, T. Kaneyasu, P. Lablanquie, F. Penent, and K. Ito, "Single and multiple auger decay processes from the $\mathrm{Ne}^{+} 1 \mathrm{~s}^{-1} 2 \mathrm{p}^{-1} \mathrm{np}$ shakeup states studied with a multielectron coincidence method," Phys. Rev. A: At., Mol., Opt. Phys., vol. 97, no. 2, p. 023405, 2018.

[58] T. Odagiri, T. Taniguchi, T. Kaneyasu, H. Tanaka, J.-I. Adachi, P. Lablanquie, and Y. Hikosaka, "Multiple auger decays of coreexcited states in $\mathrm{N}_{2}$," J. Chem. Phys., vol. 152, no. 12 , p. $124301,2020$.

[59] O. Takahashi, M. Tashiro, M. Ehara, K. Yamasaki, and K. Ueda, "Theoretical molecular double-core-hole spectroscopy of nucleobases," $J$. Phys. Chem. A, vol. 115, no. 44, pp. 12070-12082, 2011.

[60] O. Takahashi, K. Yamasaki, S.-I. Nagaoka, and K. Ueda, "Molecular double core hole electron spectroscopy of large molecules for probing molecular size: A series of bridged trihalosilyltrimethylsilyl molecules," Chem. Phys. Lett., vol. 518, pp. 44-48, 2011.

[61] O. Takahashi, N. V. Kryzhevoi, and K. Ueda, "Probing chemical environment with molecular double core-hole electron spectroscopy," J. Electron Spectrosc. Relat. Phenom., vol. 204, pp. 290 302, 2015.

[62] I. Corral, J. González-Vázquez, and F. Martín, "Potential energy surfaces of core-hole and shakeup states for dissociative ionization studies," $J$. Chem. Theory Comput., vol. 13, no. 4, pp. 17231736, 2017.

[63] J. J. Pireaux, S. Svensson, E. Basilier, P.A. Malmqvist, U. Gelius, R. Caudano, and K. Siegbahn, "Core-electron relaxation energies 
and valence-band formation of linear alkanes studied in the gas phase by means of electron spectroscopy," Phys. Rev. A: At., Mol., Opt. Phys., vol. 14, no. 6, pp. 2133-2145, 1976.

[64] J. Schirmer, G. Angonoa, S. Svensson, D. Nordfors, and U. Gelius, "High-energy photoelectron C 1 s and $\mathrm{O} 1$ s shake-up spectra of CO," J. Phys. B, vol. 20, no. 22, pp. 6031-6040, 1987.

[65] T. H. Dunning Jr., "Gaussian basis sets for use in correlated molecular calculations. I. The atoms boron through neon and hydrogen," J. Chem. Phys., vol. 90, no. 2, pp. 1007-1023, 1989.

[66] R. A. Kendall, T. H. Dunning Jr., and R. J. Harrison, "Electron affinities of the first-row atoms revisited. systematic basis sets and wave functions," J. Chem. Phys., vol. 96, no. 9, pp. 6796-6806,
1992.

[67] M. W. Schmidt, K. K. Baldridge, J. A. Boatz, S. T. Elbert, M. S. Gordon, J. H. Jensen, S. Koseki, N. Matsunaga, K. A. Nguyen, S. Su, T. L. Windus, M. Dupuis, and A. Montgomery Jr., "General atomic and molecular electronic structure system," J. Comput. Chem., vol. 14, no. 11, pp. 1347-1363, 1993.

[68] This approximation comes from considering constant $(i)$ the two electron dipole term in the regime were the correlation between the two ejected electron and the $N-2$ bound electrons is very weak (ii) the density of continuum states for photon energy far above the double core ionization potential. 